\title{
Blue rubber bleb nevus syndrome: a rare cause of chronic gastrointestinal bleed in adults
}

\author{
Gayatri Amit Deshpande ${ }^{1}$, MS, Inian Samarasam ${ }^{1}$, MS, FRCS, Sam Varghese George $^{1}$, MS, Sudhakar $\underline{\text { Chandran }}{ }^{1}$, MS
}

\begin{abstract}
Blue rubber bleb nevus syndrome (BRBNS) is a rare condition characterised by venous malformations in the skin, gastrointestinal tract and other parts of the body. Its presentation is usually sporadic, although cases of autosomal dominant inheritance have been reported. Usually seen in children, BRBNS presentation in adults is rare. Symptoms at presentation depend on the organs involved; patients with BRBNS may present with acute or chronic gastrointestinal bleed. We herein report a rare presentation of BRBNS in an adult who suffered from intermittent abdominal pain and melaena for three years. Contrast-enhanced computed tomography revealed a jejunojejunal intussusception with a vascular malformation as the lead point. The patient underwent laparotomy with resection of the intussuscepted bowel segment. Recovery was uneventful. In spite of a wide range of therapeutic options for the management of BRBNS described in the literature, the efficacy of those available therapies, including surgical excision, is not well established.
\end{abstract}

Keywords: blue rubber bleb nevus syndrome, chronic gastrointestinal bleed, intussusception

\section{INTRODUCTION}

First described by William Bean in 1958, ${ }^{(1)}$ blue rubber bleb nevus syndrome (BRBNS) is a rare condition characterised by venous malformations in the skin, gastrointestinal tract and other parts of the body. Its presentation is usually sporadic, but cases of autosomal dominant inheritance have also been reported.(2) BRBNS is usually seen in young children, and presentation in adults is rare. ${ }^{(3)}$ The skin and gastrointestinal tract are the most commonly involved, and symptoms at presentation vary according to the organ affected. Patients may present with acute gastrointestinal haemorrhage (in the form of haematemesis, melaena, or haematochezia) or chronic gastrointestinal bleed (in the form of iron deficiency anaemia). A wide range of surgical and nonsurgical modalities for the management of BRBNS have been described in the literature. However, the efficacy of the available therapies, including surgical excision, is not well established. We herein report a very rare case of BRBNS in an adult, who was successfully treated via surgical resection.

\section{CASE REPORT}

A 40-year-old man presented to our outpatient surgical department with symptoms of tiredness and easy fatiguability. The patient had no significant medical history apart from intermittent abdominal pain and melaena for three years. He appeared pale at presentation, but was haemodynamically stable, with a haemoglobin level of $6.7 \mathrm{~g} / \mathrm{dL}$. The rest of the physical examination was unremarkable. Oesophagogastroduodenoscopy and colonoscopy did not reveal the source of bleed. Contrast-enhanced computed tomography (CT) of the abdomen was performed to rule out other possible causes of bleed, showing a spectrum of findings, including a jejunojejunal intussusception with a probable vascular malformation as a lead point (Fig. 1), and the presence of multiple enhancing soft tissue density lesions in the subcutaneous plane of

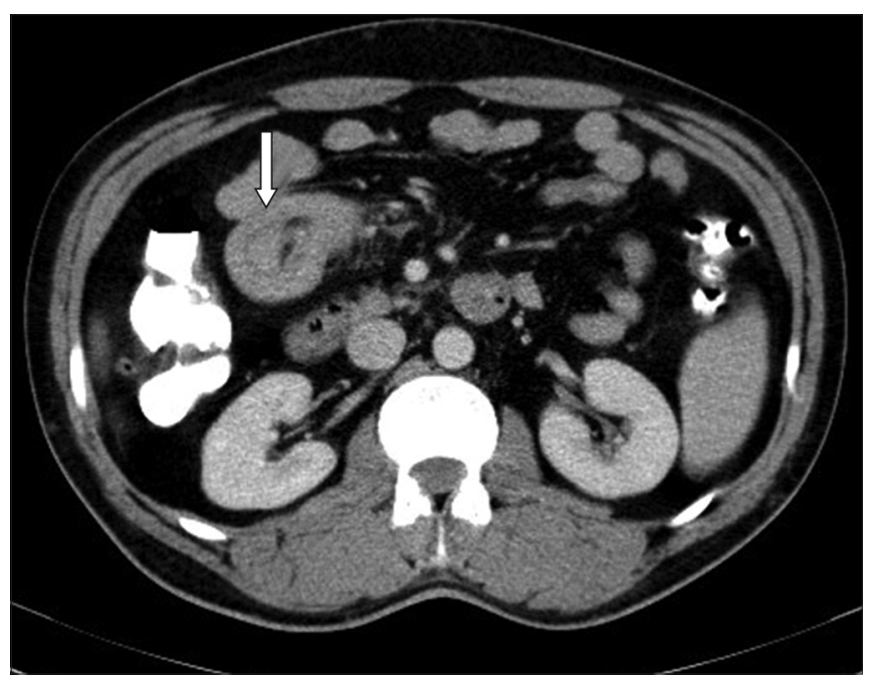

Fig. 1 CT of the abdomen reveals a jejunojejunal intussusception with a probable vascular malformation (arrow) as a lead point.

the anterior abdominal wall, which is suggestive of subcutaneous haemangioma and multiple hepatic haemangiomas (Fig. 2).

The patient underwent laparotomy with resection of the intussuscepted small bowel segment. The resected bowel revealed a polyp with superficial ulceration (Fig. 3) as a possible source of the bleed, and the lead point of the intussusception. Microscopic examination of the resected polyp showed dilated venous channels in the mucosa and submucosa. This spectrum of clinical, radiological and pathological findings is consistent with BRBNS.

\section{DISCUSSION}

Gastrointestinal lesions in BRBNS typically present as chronic continual bleed, requiring lifelong iron supplements or repeated blood transfusions. Very rarely do they present as overt bleeding with intussusception, as was seen in our patient. ${ }^{(3)}$ Upper and lower gastrointestinal endoscopy, contrast-enhanced CT of the abdomen,

${ }_{1}^{1}$ Department of General Surgery, Upper Gastrointestinal Surgery Unit, Christian Medical College and Hospital, Vellore, Tamil Nadu, India

Correspondence: Dr Gayatri Amit Deshpande, Assistant Professor, Department of General Surgery, Upper Gastrointestinal Surgery Unit, Christian Medical College and Hospital, Vellore, Tamil Nadu, India, 632 004. gayatrisabne@rediffmail.com 


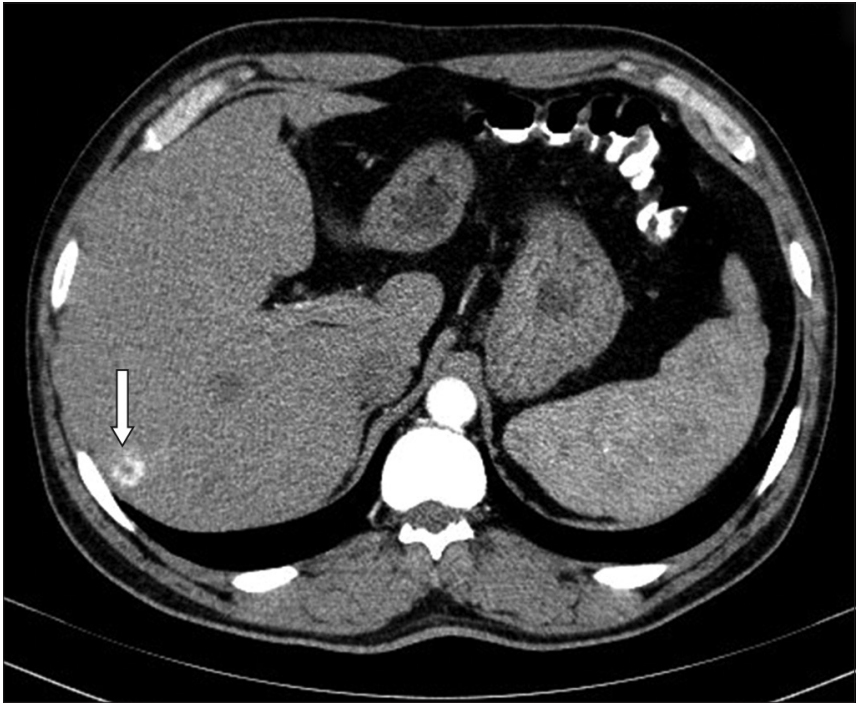

Fig. 2 CT of the abdomen reveals a hepatic haemangioma (arrow).

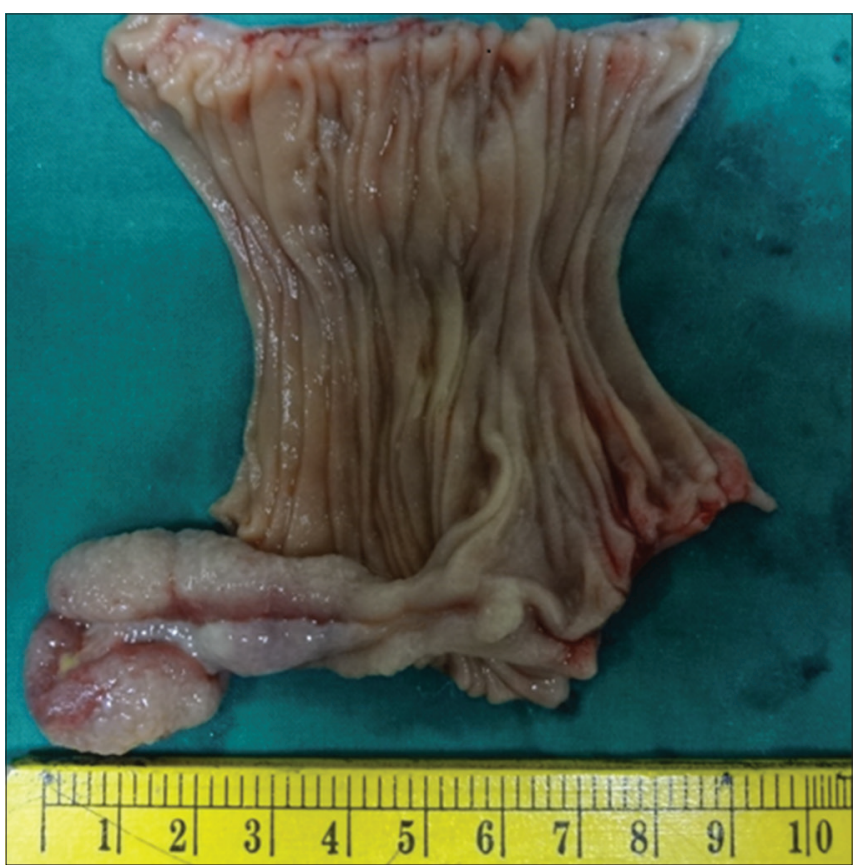

Fig. 3 Photograph shows the surgical specimen of the resected jejunum revealing a pedunculated polyp as the lead point of intussusception.

and mesenteric angiography are the recommended diagnostic modalities for BRBNS. As part of the evaluation, gastrointestinal endoscopy (oesophagogastroduodenoscopy and colonoscopy) is the first investigation of choice, and may help in localising and detecting the extent of the disease. However, in cases with a small bowel lesion, as in our patient, gastrointestinal endoscopy may fail to locate the site of bleed. In such cases, contrast-enhanced $\mathrm{CT}$ of the abdomen may be helpful in the diagnosis.

The medical literature on the treatment of BRBNS is currently limited to case reports only. The treatment of BRBNS depends on the organ involved and the symptoms at presentation, while the management of gastrointestinal lesions depends on the extent of involvement of the bowel and the severity of the bleed at presentation. In patients with chronic minimal bleed, various antiangiogenic agents (e.g. corticosteroids, interferon-alpha, gamma globulins and octreotide) have been tried, and there has been response in some patients. ${ }^{(4-6)}$ However, the long-term benefits of pharmacotherapy are still controversial. A case series by Gonzalez et al found that octreotide decreased the need for blood transfusions; ${ }^{(7)}$ the possible mechanism proposed by the authors acts by reducing blood loss from the venous malformations through a reduction in splanchnic blood flow. However, the direct effect of octreotide on venous malformations cannot be ruled out.

Gastrointestinal lesions can also be treated endoscopically, such as via endoscopic laser photocoagulation and sclerotherapy, which have been reported in the literature. ${ }^{(8)}$ For pedunculated lesions, endoscopic removal and band ligation may be attempted. The role of surgical resection in the management of gastrointestinal lesions has been controversial because of the belief that the lesions would recur after excision. ${ }^{(9)}$ In the largest case series on BRBNS to date, Fishman et al described ten patients with BRBNS who were successfully treated surgically. ${ }^{(10)}$ In their series, ${ }^{(10)}$ patients were managed with a combination of surgical techniques, which included wedge resection, polypectomy, suture ligation, segmental bowel resection and band ligation. In our patient, the small bowel intussusception was the cause of the intermittent abdominal pain, and the ulceration over the vascular polyp was the cause of the chronic gastrointestinal bleed. Hence, a segmental bowel resection bowel was warranted.

In conclusion, BRBNS is a rare surgical entity, and a wide range of therapeutic options including medical management, endoscopic therapies and surgical excision has been described. Since this syndrome is commonly associated with either occult or overt gastrointestinal bleed, it should be considered in any patient investigated for gastrointestinal bleed, as it will help in appropriate diagnosis and adequate management.

\section{REFERENCES}

1. Bean WB. Blue rubber-bleb nevi of the skin and gastrointestinal tract. In: Bean WB. Vascular Spiders and Related Lesions of the Skin. Springfield, IL: Charles C Thomas, 1958: 17-185.

2. Gallione CJ, Pasyk KA, Boon LM, et al. A gene for familial venous malformations maps to chromosome $9 \mathrm{p}$ in a second large kindred. J Med Genet 1995; 32:197-9.

3. Lee C, Debnath D, Whitburn T, Farrugia M, Gonzalez F. Synchronous multiple small bowel intussusceptions in an adult with blue rubber bleb naevus syndrome: Report of a case and review of literature. World J Emerg Surg 2008; 3:3.

4. Dieckmann K, Maurage C, Faure N, et al. Combined laser-steroid therapy in blue rubber bleb nevus syndrome: case report and review of the literature. Eur J Pediatr Surg 1994; 4:372-4.

5. Boente MC, Cordisco MR, Frontini MV, Asial RA. Blue rubber bleb nevus (Bean Syndrome): evolution of four cases and clinical response to pharmacologic agents. Pediatr Dermatol 1999; 16:222-7.

6. Aihara M, Konuma $\mathrm{Y}$, Okawa $\mathrm{K}$, et al. Blue rubber bleb nevus syndrome with disseminated intravascular coagulation and thrombocytopenia: successful treatment with high-dose intravenous gammaglobulin. Tohoku J Exp Med 1991; 163:111-7.

7. Gonzalez D, Elizondo BJ, Haslag S, et al. Chronic subcutaneous octreotide decreases gastrointestinal blood loss in blue rubber-bleb nevus syndrome. J Pediatr Gastroenterol Nutr 2001; 33:183-8.

8. Dwivedi M, Misra SP. Blue rubber bleb nevus syndrome causing upper GI hemorrhage: a novel management approach and review. Gastrointest Endosc 2002; 55:943-6.

9. Sandhu KS, Cohen H, Radin R, Buck FS. Blue rubber bleb nevus syndrome presenting with recurrences. Dig Dis Sci 1987; 32:214-9.

10. Fishman SJ, Smithers CJ, Folkman J, et al. Blue rubber bleb nevus syndrome: surgical eradication of gastrointestinal bleeding. Ann Surg 2005; 241:523-8. 